\title{
Soft tissue
}

\section{Presurgical imaging of the head}

\section{Tim Trevail}

Before choosing the best imaging modality for your patient prior to surgery, you should have an understanding of the strengths and limitations of the imaging modalities available for imaging the head. The owner's budget will also have a role to play in the choice of modality and the cost to benefit ratio will also have to be weighed in.

- Radiographs - low cost compared to advanced imaging modalities but with poor soft tissue contrast and lots of superimposition making evaluation of lesions challenging. The margins of neoplastic processes can be extremely difficult to assess on radiographs. Unless extremely enlarged, radiographs will provide no information on local lymph nodes, but thoracic staging and elimination of concurrent thoracic pathologies can be performed

- Ultrasonography - again, relatively low cost compared with advanced imaging modalities. Ultrasonography offers excellent soft tissue contrast and resolution, especially with mid-level to high-end machines. Ultrasonography of the head is limited to the superficial structures and predominantly is used ventrally to assess the draining lymph nodes of the head, the salivary glands and the tongue. Ultrasonography is an excellent modality to use to guide for tissue sampling, and is routinely used both for fine-needle aspiration (FNA) and core-needle biopsy procedures of lesions of the head and also of the draining lymph nodes

- High-field magnetic resonance imaging (MRI) - a high-cost option, with interpretation being more challenging in general than other imaging modalities. MRI has superb soft tissue contrast and resolution, being challenged only by high-end ultrasound machines. MRI is the most accurate for assessing tumour margins, although is known to slightly overestimate margins due to perilesional oedema. Isotropic three-dimensional (3D) volumetric sequences enable reconstruction in any plane, without loss of resolution, allowing for presurgical planning

- Computed tomography (CT) - again, a high-cost option, although cheaper than MRI. CT lends itself perfectly to imaging of the head due to its excellent bone detail being perfectly suited to evaluate for bone infiltration. Although excellent for assessing tumour margins, especially using a combination of bone reconstructions and soft tissue reconstructions post contrast administration, $\mathrm{CT}$ generally slightly underestimates margins. With submillimetre slice thickness, the ability to generate high-quality multi-planar reconstruction images in any plane and also to generate 3D images, CT is perfectly suited to surgical planning. In addition, due to its excellent thoracic and abdominal imaging capabilities, CT is perfectly suited to assessing both local and distant metastatic disease under the same study.

Once the images are acquired, it is imperative they are viewed in an appropriate environment. For advanced imaging modalities, the use of imaging viewing software such as Osirix is imperative to garner all the necessary information prior to surgery.

Through a series of case-based examples, this lecture will outline the benefits and limitations of each imaging modality with respect to presurgical planning of the head.

\section{KEY LEARNING OBJECTIVES}

- Understand the limitations and strengths of the various imaging modalities available for imaging the head of cats and dogs

- Understand the optimum methods for evaluating gross tumour margins for surgical planning of neoplasms of the head

- Understand the best imaging modalities for assessing for metastatic disease, especially for the local draining lymph nodes

\section{MULTIPLE CHOICE QUESTIONS}

1. Which of the following imaging modalities is best suited to assess for bone involvement associated with an acanthomatous ameloblastoma?
(A) Radiography
(B) Nuclear scintigraphy
(C) Ultrasonography
(D) $\mathrm{CT}$

2. Which of the following radiographic projections is best for assessing the tympanic bullae?
(A) Laterolateral
(B) Rostrocaudal open mouth
(C) Dorsoventral
(D) Lateral oblique

3. What is the most accurate way to assess for metastatic disease in the mandibular lymph nodes from a mast cell tumour of the nasal planum?
(A) $\mathrm{CT}$
(B) MRI
(C) Ultrasonography
(D) FNAs for cytology 